\title{
Identification of a novel mevalonate kinase gene mutation in combination with the common $M V K$ V377I substitution and the low-penetrance TNFRSF1A R92Q mutation
}

\author{
Florian Hoffmann*,1, Peter Lohse ${ }^{2}$, Silvia Stojanov ${ }^{1}$, Yoon S Shin ${ }^{3}$, Ellen D Renner ${ }^{1}$, Anja \\ Kéry $^{1}$, Stephanie Zellerer ${ }^{1}$ and Bernd H Belohradsky ${ }^{1}$

\footnotetext{
${ }^{1}$ Department of Infectious Diseases and Immunology, Children's Hospital, University of Munich, Germany;

${ }^{2}$ Department of Clinical Chemistry - Großhadern, University of Munich, Germany; ${ }^{3}$ Department of Metabolic Diseases, Children's Hospital, University of Munich, Germany
}

The hyperimmunoglobulinemia D and periodic fever syndrome (HIDS) is an autosomal recessively inherited autoinflammatory disease caused by mutations in the mevalonate kinase (MVK) gene on chromosome 12q24, which lead to a depressed enzymatic activity of mevalonate kinase (MK). TNFreceptor associated periodic syndrome (TRAPS), on the other hand, is the most frequent autosomal dominantly inherited periodic fever syndrome due to mutations in exons 2-4 and 6 of the TNFRSF1A gene on chromosome 12p13.2. We describe a girl with heterozygosity for the common MVK V377I mutation and for a novel $\mathrm{T}_{1132} \rightarrow \mathrm{C}$ transition, leading to the exchange of serine (TCC) by proline (CCC) at amino-acid position 378. Interestingly, our patient presented only with mild clinical features typical of HIDS and slightly increased immunoglobulin $\mathrm{D}$ levels, but a distinctly diminished MK activity. The girl was also heterozygous for the TNFRSF1A R92Q low-penetrance mutation, which may have significant proinflammatory effects. However, at the time of presentation, the patient had no TRAPS-associated symptoms.

European Journal of Human Genetics (2005) 13, 510-512. doi:10.1038/sj.ejhg.5201352

Publlished online 22 December 2004

Keywords: hereditary periodic fever syndromes; hyperimmunoglobulinemia D and periodic fever syndrome; HIDS; tumor necrosis factor receptor-associated periodic syndrome; TRAPS

\section{Introduction}

Hereditary periodic fever syndromes are characterized by recurrent fever episodes associated with multisystemic inflammation. Several distinct diseases have been defined by molecular genetic analyses, two of which are the

\footnotetext{
*Correspondence: Dr F Hoffmann, Department of Infectious Diseases and Immunology, Children's Hospital, Ludwig-Maximilians-University, Lindwurmstr. 4, Munich D-80337, Germany. Tel.: +49 895160 3931; Fax: +49 895160 3964; E-mail: florian.hoffmann@med.uni-muenchen.de Received 27 July 2004; revised 4 November 2004; accepted 11 November 2004
}

hyperimmunoglobulinemia $\mathrm{D}$ and periodic fever syndrome (HIDS; MIM no. 260920) and the tumor necrosis factor receptor-associated periodic syndrome (TRAPS; MIM no. 142680).

In HIDS patients, febrile episodes last about 3 to 7 days, recur every 4 to 8 weeks, and are accompanied, in almost all cases, by persistently high serum immunoglobulin D (IgD) levels $(>100 \mathrm{IU} / \mathrm{ml})$ and sometimes elevated $\operatorname{IgA}{ }^{1}$ Other typical symptoms comprise swelling of the cervical lymph nodes, chills, headache, abdominal pain, vomiting, diarrhea, arthralgia, arthritis, skin rash, splenomegaly, and/ or hepatomegaly. 1 
HIDS is an autosomal recessively inherited autoinflammatory disorder caused by mutations in the mevalonate kinase $(M V K)$ gene on chromosome 12q24, which lead to a depressed enzymatic activity of mevalonate kinase (MK). ${ }^{2,3}$ More than 30 mutations have been reported in exons 2-11 of the $M V K$ gene. ${ }^{4}$ The vast majority are missense mutations. Most probands are compound heterozygotes for V377I and another missense mutation, which usually results in a nonfunctional enzyme. ${ }^{5}$

TRAPS, in contrast, is the most frequent autosomal dominantly inherited periodic fever syndrome. Attacks are associated with severe abdominal pain, localized myalgia, painful migratory erythematous skin rash, conjunctivitis, and/or periorbital edema. TRAPS is caused by mutations in exons 2-4 and 6 of the TNFRSF1A gene on chromosome $12 \mathrm{p} 13.2$, which encodes the $55-\mathrm{kDa}$ receptor for tumor necrosis factor. ${ }^{6}$ Up to now, more than 40 mutations have been identified. ${ }^{4,7}$

\section{Patients and methods \\ Patient}

We describe a 7-year-old German female, who initially was referred to our department with recurrent viral infections and fever up to $41^{\circ} \mathrm{C}$ since the age of 3 months. Attacks lasted 3 to 7 days, were accompanied by increases of inflammatory parameters and interrupted by symptomfree intervals of 2-12 weeks duration. Within the last year, these febrile episodes became shorter and were less frequent.

Between fever attacks, the girl had slightly elevated serum IgD values of 129 and $127 \mathrm{IU} / \mathrm{ml}$ (normal value: $<100 \mathrm{IU} / \mathrm{ml}$ ), while IgA levels were within the normal range. This prompted us to determine the MK activity in peripheral blood leukocytes, which was very low with $0.02 \mathrm{nmol} / \mathrm{min} / \mathrm{mg}$ protein (normal range: $0.4-1.0$ ).

Careful questioning revealed chills, vomiting, arthralgias, and cervical as well as inguinal lymphadenopathy as symptoms, which were regularly associated with the fever attacks. Occasionally, abdominal pain and diarrhea were also present. Myalgia, conjunctivitis, periorbital edema, arthritis, or rash, in contrast, were never observed. Mevalonic aciduria-specific neurologic signs were also absent.

The patient is thriving normally and shows no signs of proteinuria. Serum amyloid A levels are within the normal range.

\section{DNA sequence analysis of the MEFV and TNFRSF1A genes}

EDTA blood samples were collected from the patient and all family members, and genomic DNA was isolated from white blood cells with the QIAamp blood mini kit (QIAGEN, Hilden, Germany). MVK and TNFRSF1A gene analyses were performed as described previously. ${ }^{8}$

\section{Analysis of leukocyte MVK activity}

MK activity was measured in leukocytes prepared from $3 \mathrm{ml}$ of peripheral EDTA blood. ${ }^{9}{ }^{14} \mathrm{C}$-mevalonate-5-phosphate was separated from the substrate ${ }^{14} \mathrm{C}$-mevalonic acid on a mini DEAE-cellulose column. The normal range of enzymatic activity is $0.4-1.0 \mathrm{nmol} / \mathrm{min} / \mathrm{mg}$ protein.

\section{Results}

DNA sequence analysis demonstrated that the girl was a compound heterozygous carrier of the most common $M V K$ missense mutation V377I/G $\mathrm{G}_{1129} \rightarrow \mathrm{A}$ and of a novel $\mathrm{T}_{1132} \rightarrow$ $\mathrm{C}$ transition, leading to the replacement of serine (TCC), residue 378 , by proline (CCC). This nucleotide substitution was also found in her asymptomatic father (IgD $4 \mathrm{IU} / \mathrm{ml})$ and in her brother (IgD not detectable), but was not present on 370 control chromosomes tested, thereby strongly supporting the assumption that the S378P exchange encoded by $M V K$ exon 11 is indeed a true mutation. Sequencing of the proband's DNA revealed, in addition, a heterozygous carrier state for the low-penetrance TNFRSF1A R92Q mutation, which was also present in her asymptomatic mother in combination with heterozygosity for the MVK V377I substitution (IgD not detectable). MK enzymatic activity in all carriers with one $M V K$ mutation was slightly diminished, irrespective of whether residue 377 or 378 was affected (mother: $0.30 \mathrm{nmol} / \mathrm{min} /$ $\mathrm{mg}$ protein; father: $0.39 \mathrm{nmol} / \mathrm{min} / \mathrm{mg}$ protein; brother: $0.32 \mathrm{nmol} / \mathrm{min} / \mathrm{mg}$ protein).

\section{Discussion}

In combination with heterozygosity for the common V377I mutation, the novel S378P substitution results in a distinctly diminished MK activity of $2-5 \%$ of healthy controls. Usually, in HIDS patients without neurological symptoms, enzymatic activity is reduced to an average of $9.2 \%(1.8-28.0 \%)$ with a standard deviation of $6 \% .{ }^{10}$ It is therefore quite surprising that our patient presented only with mild clinical features typical of HIDS (cervical lymphadenopathy, mild abdominal complaints, arthralgia, chills) and slightly increased IgD levels. This illustrates the wide variability in the clinical presentation of HIDS and the absence of a clear relationship between genotype, residual MK activity, and clinical features, as has been observed already by others. ${ }^{11}$

The TNFRSF1A R92Q substitution, on the other hand, is a mutation with incomplete penetrance, present in both symptomatic TRAPS patients as well as in asymptomatic controls and occurring with an allele frequency of $\sim 1 \%$ in Irish and North American control populations. ${ }^{12}$ Heterozygosity for this low-penetrance mutation is expected to augment the intensity of the autoinflammatory response, ${ }^{13,14}$ thereby resulting in a more severe phenotype, especially as HIDS has been shown to be associated with increased TNF and soluble TNF receptor levels. ${ }^{15}$ From a 
clinical standpoint, however, this appears not to be the case in our proband.

Previously, only one other patient with mutations in two autoinflammatory genes has been described. The 3-yearold boy carried two MVK mutations (G211A and V377I) and the TNFRSF1A low-penetrance P46L substitution. ${ }^{16}$ Similar to our girl, he did not show any TRAPS-typical symptoms; however, serum TNFRSF1A levels were decreased, and he responded at least partially to TNF receptor blockade with etanercept, suggesting that the P46L mutant contributed to the phenotype. In our patient, treatment with etanercept has not yet been tried for two reasons: (1) the girl responds well to corticosteroids and (2) R92Q seems not to be associated with impaired TNF receptor shedding. ${ }^{12}$

\section{References}

1 Drenth JP, Haagsma CJ, van der Meer JW: Hyperimmunglobulinemia $\mathrm{D}$ and periodic fever syndrome: the clinical spectrum in a series of 50 patients. International Hyper-IgD Study Group. Medicine (Baltimore) 1994; 73: 133-144

2 Drenth JPH, Cuisset L, Grateau G et al: Mutations in the gene encoding mevalonate kinase cause hyper-IgD and periodic fever syndrome. Nat Genet 1999; 22: 178-181.

3 Houten SM, Kuis W, Duran M et al: Mutations in MVK, encoding mevalonate kinase, cause hyperimmunglobulinaemia $\mathrm{D}$ and periodic fever syndrome. Nat Genet 1999; 22: 175-177.

4 Sarrauste de Menthiere C, Terriere S, Pugnere D et al: INFEVERS: the Registry for FMF and hereditary inflammatory disorders mutations. Nucleic Acids Res 2003; 31: 282-285.

5 Drenth JP, van der Meer JW: Hereditary periodic fever. $N$ Engl J Med 2001; 345: 1748-1757.

6 McDermott MF, Aksentijevich I, Galon J et al: Germline mutations in the extracellular domains of the $55 \mathrm{kDa}$ TNF receptor (TNF-R1) define a family of dominantly inherited autoinflammatory syndromes. Cell 1999; 97: 133-144.
7 Kusuhara K, Nomura A, Nakao F, Hara T: Tumor necrosis factor receptor-associated periodic fever syndrome with a novel mutation in the TNFRSF1A gene in a Japanese family. Eur J Pediatr 2004; 163: 30-32.

8 Stojanov S, Lohse P, Lohse P et al: Molecular analysis of the MVK and TNFRSF1A genes in patients with a clinical presentation typical of the hyperimmunoglobulinemia $\mathrm{D}$ with periodic fever syndrome: a low-penetrance TNFRSF1A variant in a heterozygous MVK carrier possibly influences the phenotype of hyperimmunoglobulinemia D with periodic fever syndrome or vice versa. Arthritis Rheum 2004; 50: 1951-1958.

9 Hoffmann GF, Brendel SU, Scharfschwerdt SR et al: Mevalonate kinase assay using DEAE-cellulose column chromatography for first-trimester prenatal diagnosis and complementation analysis in mevalonic aciduria. J Inher Metab Dis 1992; 15: 738-746.

10 Cuisset L, Drenth JPH, Simon A et al: Molecular analysis of MVK mutations and enzymatic activity in hyper-IgD and periodic fever syndrome. Eur J Hum Gen 2001; 9: 260-266.

11 Frenkel J, Houten SM, Waterham HR et al: Clinical and molecular variability in childhood periodic fever with hyperimmunoglobuliaemia D. Rheumatology 2001; 40: 579-584.

12 Aksentijevich I, Galon J, Soares M et al: The tumor-necrosis-factor receptor-associated periodic syndrome: new mutations in TNFRSF1A, ancestral origins, genotype-phenotype studies, and evidence for further genetic heterogeneity of periodic fevers. Am J Hum Genet 2001; 69: 301-314.

13 Aganna E, Hammond L, Hawkins PN et al: Heterogeneity among patients with tumor necorsis factor receptor-associated periodic syndromes phenotypes. Arthritis Rheum 2003; 48: 2632-2644.

14 Aganna E, Hawkins PN, Ozen S et al: Allelic variants in genes associated with hereditary periodic fever syndromes as susceptibility factors for reactive systemic AA amyloidosis. Genes Immun 2004; 5: 289-293.

15 Aganna E, Aksentijevich I, Hitman GA et al: Tumor necrosis factor receptor-associated periodic fever syndrome (TRAPS) in a Dutch family: evidence for a TNFRSF1A mutation with reduced penetrance. Eur J Hum Genet 2001; 9: 63-66.

16 Arkwright PD, McDermott MF, Houten SM et al: Hyper IgD syndrome (HIDS) associated with in vitro evidence of defective monocyte TNFRSF1A shedding and partial response to TNF receptor blockade with etanercept. Clin Exp Immunol 2002; 130: $484-488$. 